\title{
Programa para la mejora de la sensibilidad (PMS) y habilidades de interacción del profesorado de Educación Infantil
}

\section{Program for sensitivity improvement (SIP) and the interaction skills of the Infant Education educator}

\author{
Alexander Barandiaran Arteaga*1 \\ abarandiaran@mondragon.edu \\ Alexander Muela Aparicio** \\ alexander.muela@ehu.es \\ Elena López de Arana Prado** \\ Elena.lopezdearana@ehu.es \\ IÑAKI LARREA HeRMIDA* \\ ilarrea@mondragon.edu \\ Agurtzane Martínez Gorrotxategi* \\ amartinez@mondragon.edu \\ *Mondragon Unibertsitatea, España \\ **Universidad del País Vasco UPV-EHU, España
}

\section{Resumen:}

La importancia del desarrollo profesional del profesorado de Educación Infantil, para satisfacer las necesidades evolutivas infantiles y garantizar una educación de calidad, ha obtenido una gran fundamentación teórica y empírica. Investigaciones recientes han puesto de manifiesto que, entre las variables que afectan al desarrollo temprano, las habilidades de interacción del docente son especialmente relevantes. Además, diversos meta-análisis recomiendan un tipo de formación centrada en el análisis de la práctica docente a través del video feedback. Sin embargo, son escasos los

\begin{abstract}
:
The relevance of professional development aimed at satisfying children's developmental needs and guaranteeing quality education has achieved a great theoretical and empirical support. According to recent research, among the variables that influence early development care takers' interaction skills are especially relevant. Furthermore, diverse meta-analysis suggest a type of training based on the analysis of practice through video-feedback. However, very few training programs have applied this methodology in Spain so far. That is why the objective of this study was to analyse
\end{abstract}

1 Dirección para correspondencia (correspondence address):

Alexander Barandiaran Arteaga. Dpto. de Innovación e Intervención en Educación Inclusiva. Facultad de Humanidades y Ciencias de la Educación. Universidad de Mondragón. Bo Dorleta, s/n. 20540, Eskoriatza, Guipúzcoa (España). 
Programa para la mejora de la sensibilidad (PMS) y habilidades de interacción del profesorado de Educación Infantil

Alexander Barandiaran Arteaga, Alexander Muela Aparicio, Elena lópez de Arana Prado, Iñaki Larrea Hermida y Agurtzane Martínez Gorrotxategl

programas de desarrollo profesional que hayan aplicado esta metodología en España. Por ello, el objetivo del presente trabajo consistió en examinar la influencia del programa para la mejora de la sensibilidad (PMS) sobre las habilidades de interacción del profesorado de Educación Infantil. En el estudio participaron 14 educadoras divididas en dos grupos. El grupo de intervención $(n=7)$, que recibió la formación, y el grupo de control $(n=7)$. Los resultados indican que, tras someterse al PMS, las educadoras muestran una mayor sensibilidad y conductas de estimulación verbal. Además, en comparación con las profesoras que no habían recibido la intervención, obtuvieron una mayor puntuación en sensibilidad y estimulación del lenguaje. Aunque en el futuro ha de replicarse el estudio con un mayor número de sujetos, los resultados obtenidos permiten afirmar que el PMS es un tipo de formación que puede resultar muy efectiva para la práctica educativa del profesorado de Educación Infantil.

\section{Palabras clave:}

Desarrollo profesional; habilidades de interacción; educación Infantil; video feedback; calidad del cuidado; estimulación verbal; práctica reflexiva. the influence the training program for sensitivity improvement (SIP) has on the interaction skills of Infant Education educators. 14 care-takers participated in the research, divided into two groups. The experimental group $(\mathrm{n}=7)$, who were trained, and the comparison or control group $(n=7)$. Results show that once the PMS training program had taken place, the educators showed greater sensitivity and verbal stimulation conducts. Besides, in comparison to the teachers who were not treated not undergo the treatment, they obtained higher scores in sensitivity and language stimulation. Despite the fact that the research should be replicated with a bigger sample in the future, the results obtained allow to state that PMS is a type of training that could be effective for Infant Education teachers' educational practice.

\section{Keywords:}

Professional development; interaction skills; preschool education; video feedback; childcare quality; verbal stimulation; teacher's reflective practice.

\section{Résumé:}

L'importance de la formation des enseignants de I'Ecole Maternelle, dont les objectifs consistent à répondre aux besoins de développement des enfants et à assurer une éducation de qualité, a permis de construire une large base théorique et empirique. Des recherches récentes ont montré que parmi les variables qui influencent sur le développement précoce, les capacités dsinteraction de liéducateur sont particulièrement importantes. En outre, diverses méta-analyses recommandent un type de formation axé sur lianalyse des pratiques dienseignement par le biais du vidéo-feedback. Cependant, il y a eu peu de programmes de formation qui aient appliqué cette méthodologie en Espagne. Par conséquent, l>objectif de cette étude a été d`examiner lsinfluence du programme de formation pour améliorer la sensibilité (PMS) sur les capacités d)interaction de lıenseignant de la maternelle. L`étude a impliqué 14 éducateurs répartis en deux groupes. Le groupe formé $(n=7)$, et le groupe témoin $(n=7)$. Les résultats indiquent que, après s'être soumis au PMS, les éducatrices montrent une plus grande sensibilité pour travailler la stimulation verbale. En outre, elles ont obtenu de meilleurs résultats en sensibilité et stimulation du langage par rapport aux enseignants qui n'ont pas participé à la formation. Bien que, dans l'avenir il faudra refaire liétude avec un plus grand nombre de sujets, les résultats confirment que le PMS est un type de formation qui peut être très efficace pour la pratique pédagogique des enseignants en Ecole Maternelle.

\section{Mots clés:}

Formation continue; capacités d'interaction; école maternelle; vidéo-feedback; qualité des soins; stimulation verbale; pratique réflexive. 
Alexander Barandiaran Arteaga, Alexander Muela Aparicio, Elena lópez de Arana Prado,

Fecha de recepción: 3-11-2014

Fecha de aceptación: 3-6-2015

\section{Introducción}

El desarrollo profesional docente hace referencia a procesos mediante los cuales el profesorado mejora su repertorio de capacidades profesionales (Marcelo y Vaillant, 2009). La importancia del desarrollo profesional del docente del ciclo de Educación Infantil, de cara a satisfacer las necesidades evolutivas infantiles y garantizar una educación de calidad, ha obtenido una gran fundamentación teórica y empírica (Clarke-Stewart y Allhusen, 2005; Fukkink y Lont, 2007; Ochaita y Espinosa, 2001; OECD, 2012; Zaslow, Anderson, Redd, Wessel, Tarullo y Burchinal, 2010). En España esto cobra una importancia considerable, puesto que los niños y las niñas ingresan cada vez más pronto en la escuela y cada vez pasan mayor tiempo en ella. A diferencia de la mayoría de los Estados miembros, en los que por lo general los niños y las niñas ingresan en la escuela a partir de los 5 años de edad, en España el 90\% de los niños y las niñas acceden a esta institución a la edad de 3 años, y casi su totalidad se encuentra matriculada a los 4 años de edad (OECD, 2013, 2014).

Según Imbermón (1994), se requiere de un desarrollo profesional de carrera que implica un aprendizaje constante durante la realización de la actividad docente. Es decir, un desarrollo profesional sustentado en la reflexión y el análisis de la práctica profesional como estrategia de trabajo conjunto, cuya repercusión alcanza tanto a la capacitación del profesorado como a la propia institución. En palabras de dicho autor, "la formación debe proponer un proceso que capacite al profesorado para aprender a aprender, pero también para aprender a desaprender en comunicación, autoanálisis y regulación propia mediante conocimientos, destrezas y actitudes a fin de desarrollar profesionales inquietos e innovadores que aprendan de sus aciertos y errores" (Imbermón, 2007, p.123) y propone el seguimiento del proceso formativo mediante la observación de compañeros o asesores, ya que garantiza el traspaso de estrategias de enseñanza de mayor complejidad (Imbermón, 2005).

A este respecto, se han llevado a cabo diversas iniciativas de desarrollo profesional basadas en el análisis de la práctica por medio del video feedback; no obstante, son escasas las propuestas que han tratado de imple- 
Programa para la mejora de la sensibilidad (PMS) y habilidades de interacción del profesorado de Educación Infantil

Alexander Barandiaran Arteaga, Alexander Muela Aparicio, Elena lópez de Arana Prado, Iñaki Larrea Hermida y Agurtzane Martínez Gorrotxategl

mentar esta metodología. Este es el punto de partida del presente artículo, que pretende examinar la influencia de un programa para la mejora de la sensibilidad (PMS) sobre las habilidades de interacción del profesorado de Educación Infantil en una muestra de la Comunidad Autónoma Vasca.

\section{El desarrollo profesional docente en la etapa de Educación Infantil}

La Educación Infantil es una fuente de beneficio para el alumnado, sus familias y la sociedad en su conjunto (Ancheta, 2012). Sin embargo, la magnitud de estos beneficios está condicionada por su calidad del cuidado estructural y de proceso (Lera, 2007; NICHD, 2005; OECD, 2012; Vandell, Belsky, Burchinal, Vandergrift y Steinberg, 2010). Numerosos estudios han puesto de manifiesto que la Educación Infantil de una alta calidad mejora el bienestar emocional, el aprendizaje y el desarrollo cognitivo infantil (Burchinal et al., 2000, 2008; De Shipper, Riksen-Walraven y Geurts, 2006; Peisner-Feinberg et al., 2001; Ruzek, Burchinal, Farkas y Duncan, 2014). Con el objetivo de consolidar estos beneficios para el desarrollo infantil, obra un compromiso internacional para incentivar políticas educativas implicadas en la alta calidad del cuidado. Por lo que recientemente se ha trazado la línea clave del desarrollo profesional del docente y sus condiciones de trabajo (OECD, 2012).

Diversos autores (Imbermon, 2007; Marcelo, 2011; Marcelo y Vaillant, 2009) han puesto de manifiesto la importancia del desarrollo profesional docente para dar respuesta a los desafíos actuales de la educación en una sociedad en permanente cambio social, económico y cultural. La educación es considerada como la variable fundamental para definir el futuro de una sociedad sustentada en el conocimiento, y los docentes son los actores fundamentales de este escenario educativo (Marcelo, 2009). En esta línea y respecto a la formación inicial del profesorado, el Ministerio de Educación y Ciencia (Orden ECI/3854/2007) ha establecido un perfil de maestro/a de Educación Infantil caracterizado por competencias profesionales que implican: el conocimiento de las características de la etapa evolutiva de 0-6 años (objetivos, contenidos curriculares, criterios de evaluación, aspectos organizativos y de funcionamiento, etc.); la promoción y la facilitación de los aprendizajes desde una perspectiva globalizadora y el diseño de espacios para tal fin; el fomento de la convi- 
Alexander Barandiaran Arteaga, Alexander Muela Aparicio, Elena lópez de Arana Prado,

vencia, la orientación a las familias y la propia reflexión sobre la práctica profesional para innovar y mejorar la labor docente. Estas competencias están ligadas a factores relacionales y metodológicos clave de la Educación Infantil (Zabalza, 1996). Entre dichos factores cabe destacar que el profesorado preste atención privilegiada a la dimensión emocional de los niños/as, emplee un lenguaje enriquecido y ofrezca atención individualizada, tanto a los menores como a sus familias. Así como, la adecuada organización de los espacios y los materiales, la evaluación y la planificación, el desarrollo y la diferenciación de actividades. A todo ello, cabría añadir la trascendencia de la capacidad introspectiva del docente en relación a su trabajo educativo (Zabalza y Zabalza, 2011).

Así pues, en la Educación Infantil, vinculado al desarrollo profesional continuo, se otorga una gran importancia a la formación de un perfil de maestro/a dotado de competencias profesionales para crear entornos pedagógicos de alta calidad del cuidado que favorezcan la consecución de los hitos evolutivos de esta etapa del desarrollo. Entornos educativos caracterizados por factores críticos, tales como la capacidad del profesorado para mostrarse sensible ante las señales y necesidades del alumnado y establecer así una relación segura con ellos. La competencia del docente para generar espacios de aprendizaje estimulantes y favorecer actividades que permitan al niño/a explorar activamente el entorno de un modo relativamente independiente, que promuevan su competencia y autoeficacia. Así como la capacidad del docente para estimular las interacciones sociales entre los niños y el uso de diversas estrategias de andamiaje (OECD, 2012; Phillips y Lowenstein, 2011).

\section{El video feedback como herramienta para la mejora de las habilidades de interacción del profesorado}

Investigaciones recientes han puesto de relieve que, entre las variables que afectan al desarrollo temprano, las habilidades de interacción del profesorado son especialmente importantes para que niños/as aborden con éxito la transición entre el contexto familiar y el escolar, así como las complejas y continuadas experiencias relacionales con otros niños, personas adultas, espacios y materiales (Barandiaran, Muela, López de Arana, Larrea y Vitoria, 2015; Belsky, 2006; Hamre, Pianta, Hatfield y Jamil, 2014; Vandell et al., 2010). 
Alexander Barandiaran Arteaga, Alexander Muela Aparicio, Elena lópez de Arana Prado, iñaki Larrea Hermida y Agurtzane Martínez Gorrotxategi

Con el objetivo de promover en el profesorado de Educación Infantil mayores habilidades de interacción, caracterizadas principalmente por la respuesta sensible y la estimulación verbal, se han diseñado diversos programas de desarrollo profesional. Estos programas tienen en común el hecho de estar orientados al análisis y mejora de la práctica docente del participante a través de la metodología del video feedback (Fukkink, Trienekens y Kramer, 2011). Esta metodología se caracteriza principalmente por el análisis de fragmentos grabados en video de las interacciones entre los niños y los educadores/as que ocurren en espacios y situaciones escolares naturales, y la discusión resultante del visionado de las imágenes entre el formador y el participante. El maestro/a tiene la oportunidad de observar su práctica desde la distancia. Puede focalizar su atención en múltiples conductas que incluyen aspectos verbales, no-verbales y paralingüísticos y permitirse un espacio para la reflexión. El video feedback desempeña una función catalizadora de la reflexión crítica y provee al participante y al formador una herramienta para iniciar un diálogo conjunto sobre la práctica escolar (Bautista, 2013; Tripp y Rich, 2012; van Vonderen, Didden y Beeking, 2012; Virmani y Ontai, 2010).

Según el meta-análisis realizado por Fukkink y Lont (2007), el desarrollo profesional centrado en el análisis de la práctica mejora la competencia docente en la calidad del cuidado $(d=0,45, D T=0,10)$ y tiene un efecto positivo en los niños $(d=0,55, D T=0,30)$. A esto se ha de añadir que en otro meta-análisis realizado por el mismo autor (Fukkink et al., 2011), se concluye que la formación del profesorado basado en el video feedback mejora las habilidades de interacción de los profesionales $(d=$ $0,40, D T=0,07)$. Así pues, con estos datos en la mano, queda ratificada, y a su vez se recomienda, el desarrollo profesional basado en el análisis de la práctica a través del video feedback, a la hora de abordar dichas competencias profesionales en el desarrollo profesional del profesorado de Educación Infantil.

A partir de estas consideraciones, se han llevado a cabo diversas iniciativas de desarrollo profesional basadas en el análisis de la práctica por medio del video feedback, y se han obtenido resultados muy positivos sobre las habilidades de interacción del profesorado de Educación Infantil (Elicker, Georgescu y Bartsch, 2008; Fukkink y Tavechhio, 2010; Pianta, Mashburn, Downer, Hamre y Justice, 2008). Por ejemplo, Fukkink y Tavechhio (2010) Ilevaron a cabo un estudio en el que participaron 92 
Alexander Barandiaran Arteaga, Alexander Muela Aparicio, Elena lópez de Arana Prado,

profesores, divididos en un grupo de intervención y un grupo de control. El grupo de intervención, compuesto por 52 profesores, recibió una formación especializada cuyo objetivo era la mejora de las capacidades de interacción del docente. Tras la aplicación del programa de formación, observaron que estos profesores/as mostraban en la práctica educativa un incremento de la sensibilidad y la estimulación verbal. Asimismo, hallaron que, en comparación con el grupo de control que no recibió la formación $(n=43)$, presentaban una mayor respuesta sensible a las señales de los niños y mayores conductas de estimulación verbal.

Resultados similares se obtuvieron en el estudio llevado a cabo por Pianta et al. (2008). Sin embargo, en este caso el grupo control recibió una formación de modalidad on-line de baja supervisión, en el que los participantes tuvieron acceso a grabaciones de ejemplos de buenas prácticas, pero no realizaron análisis alguno de su propia práctica. Los docentes del grupo de tratamiento, que recibieron una formación añadida focalizada en el análisis de su propia práctica a partir del video feedback, presentaron una mayor sensibilidad, una mayor cantidad y calidad del uso de técnicas de estimulación del lenguaje y aplicación de un formato pedagógico en el proceso de enseñanza-aprendizaje.

\section{El Programa para la Mejora de la Sensibilidad (PMS)}

El desarrollo profesional docente en España está orientado a las necesidades de los docentes, a la mejora y a la innovación escolar (Marcelo y Vaillant, 2009). No obstante, a pesar de la repercusión y prestigio internacional de los estudios descritos anteriormente y la citada recomendación para promover un desarrollo profesional centrado en el análisis de la práctica a través del video feedback (Fukkink et al., 2011), todavía son escasos los programas de formación encaminados a la mejora de las habilidades de interacción a través de dicha metodología. Además, aunque muchos de los estudios realizados en torno al desarrollo profesional del profesorado de Educación Infantil enfatizan la necesidad de centrarse en el análisis de la práctica docente (Calderón y Martínez, 2014; Manzanares y Galván-Bovaira, 2012), no están explícitamente dirigidos a la mejora de la sensibilidad y la competencia de la estimulación verbal. Por otra parte, no conocemos estudios realizados en nuestro país que hayan evaluado la influencia de un programa de formación de estas características. 
Alexander Barandiaran Arteaga, Alexander Muela Aparicio, Elena lópez de Arana Prado,

El Programa para la Mejora de la Sensibilidad (PMS) que se presenta en este artículo, tiene su origen en el sistema de intervención de retroalimentación mediante videograbaciones para promover la crianza positiva en el ámbito familiar (VIPP, Juffer, Bakermans-Kranenburg y Van IJzendoorn, 2008). Se basa en la teoría del apego (Bowlby, 1969), que destaca la importancia de la respuesta sensible de los cuidadores frente a las necesidades emocionales de sus hijos/as. El objetivo principal del PMS es incrementar la calidad del cuidado del maestro/a a través de la mejora de la respuesta sensible y una mayor efectividad en cubrir las necesidades de cada niño/a, en el contexto escolar de atención grupal.

En primer lugar, el maestro/a y el alumnado son grabados en video en una actividad escolar cotidiana durante 30-40 minutos. En el período que trascurre entre la grabación y la sesión de formación presencial (una semana), el formador analiza la grabación y prepara sus comentarios sobre la conducta del alumnado y las reacciones del docente. En la sesión de formación presencial, de aproximadamente 2 horas de duración, el maestro/a y el formador, conjuntamente visionan y discuten cada uno de los fragmentos seleccionados. El formador concentra toda la atención en las interacciones positivas y exitosas del maestro/a. La intervención trata de que el profesorado se perciba competente en el cuidado sensible, capaz de satisfacer las necesidades de apego y exploración de los niños y las niñas. El PMS se centra en cuatro contenidos fundamentales que son tratados sucesivamente: (a) La exploración y el apego: el objetivo del primer tema es comprobar cuándo y cómo el menor está demandando seguridad y exploración. Lo que se pretende es entrenar a los maestros/ as en la observación de estas conductas para que seguidamente puedan responder adecuadamente. (b) El "dar voz al niño": se fomenta la correcta interpretación de las señales sutiles de los niños/as mediante verbalizaciones de sus expresiones faciales y las manifestaciones no verbales que se recogen en las videograbaciones; (c) la "cadena de sensibilidad": se explica la importancia de dar una respuesta rápida y ajustada a las señales de los niños/as (señal del niño/a- respuesta del maestro/a- reacción del niño/a) y (d) el compartir emociones: se muestra y promueve la sintonía afectiva del maestro/a con las emociones positivas y negativas de los niños/as. 
Alexander Barandiaran Arteaga, Alexander Muela Aparicio, Elena lópez de Arana Prado, iñaki Larrea Hermida y Agurtzane Martínez Gorrotxategi

\section{Diseño y Metodología}

El objetivo del presente estudio radica en examinar la influencia de un programa de desarrollo profesional para la mejora de la sensibilidad (PMS), basado en el video feedback y adaptado para su aplicación en la cultura española, sobre las habilidades de interacción en la práctica educativa. Además, se pretende conocer si existen diferencias respecto a estas variables entre el profesorado que participa en esta formación y los docentes que no son sometidos al PMS.

Se espera que, tras someterse al PMS, los maestros/as presenten una mayor sensibilidad y conductas de estimulación verbal. Además, se espera que tales educadores/as obtengan una mayor puntuación que los educadores/as no sometidos al programa de formación, en las variables de sensibilidad y estimulación verbal.

Para alcanzar estos objetivos se planteó un diseño de grupo control no equivalente, con una medida pretest y una evaluación postratamiento. De esta forma, se examinó la influencia del PMS sobre las variables criterio tanto desde una aproximación intrasujeto (comparación entre las medidas pretratamiento y postratamiento en el grupo de tratamiento) como intersujetos (comparación entre las medidas postratamiento del grupo de tratamiento y del grupo control). La elección de este diseño se basó en criterios utilizados en estudios similares que han implementado el video feedback en la formación de los docentes con objeto de mejorar las habilidades de interacción (véase p, ej., Fukkink y Tavechhio, 2010).

\section{Participantes}

En el estudio participaron 7 centros escolares pertenecientes a la red privada concertada de la Comunidad Autónoma del País Vasco, los cuales fueron seleccionados sobre la base de su disponibilidad para el estudio. La muestra estuvo formada por 14 educadoras del aula de 2-3 años de Educación Infantil divididas en dos grupos. Tanto el grupo de tratamiento como el grupo control, uno por cada centro escolar, estaban constituidos por 7 educadoras. Las educadoras del grupo de tratamiento se ofrecieron voluntarias para participar en el programa de formación.

Con el objetivo de que ambos grupos fuesen comparables, se utilizaron los siguientes criterios de emparejamiento: presentar una experiencia 
Programa para la mejora de la sensibilidad (PMS) y habilidades de interacción del profesorado de Educación Infantil

Alexander Barandiaran Arteaga, Alexander Muela Aparicio, Elena lópez de Arana Prado,

Iñaki Larrea Hermida y Agurtzane Martínez Gorrotxategl

profesional similar, presentar la misma formación académica, tener una edad similar (no diferencias mayores de 10 años) y tener el mismo sexo. Además, cabe señalar que los sujetos pertenecientes al grupo control realizaban, excepto en lo que se refiere al programa de formación con el PMS, las mismas actividades y rutinas que el grupo de intervención.

En la tabla 1 se presentan los datos sociodemográficos relativos a la muestra.

Tabla 1. Datos sociodemográficos de la muestra.

\begin{tabular}{|c|c|c|c|c|c|c|}
\hline & \multicolumn{2}{|c|}{ Muestra Total } & \multicolumn{2}{|c|}{$\begin{array}{l}\text { Grupo trata- } \\
\text { miento }\end{array}$} & \multicolumn{2}{|c|}{ Grupo control } \\
\hline & $n$ & Porcentaje & $n$ & $\begin{array}{l}\text { Porcen- } \\
\text { taje }\end{array}$ & $n$ & $\begin{array}{l}\text { Porcen- } \\
\text { taje }\end{array}$ \\
\hline \multicolumn{7}{|l|}{ Titularidad del centro } \\
\hline Público & 0 & $0 \%$ & 0 & $0 \%$ & 0 & $0 \%$ \\
\hline Privado & 14 & $100 \%$ & 7 & $100 \%$ & 7 & $100 \%$ \\
\hline Total & 14 & $100 \%$ & 7 & $100 \%$ & 7 & $100 \%$ \\
\hline \multicolumn{7}{|l|}{ Ubicación del centro } \\
\hline Zona Rural & 0 & $0 \%$ & 0 & $0 \%$ & 0 & $0 \%$ \\
\hline Zona Urbana & 14 & $100 \%$ & 7 & $100 \%$ & 7 & $100 \%$ \\
\hline Total & 14 & $100 \%$ & 7 & $100 \%$ & 7 & $100 \%$ \\
\hline \multicolumn{7}{|l|}{ Género participantes } \\
\hline Varón & 0 & $0 \%$ & 0 & $0 \%$ & 0 & $0 \%$ \\
\hline Mujer & 14 & $100 \%$ & 7 & $100 \%$ & 7 & $100 \%$ \\
\hline \multicolumn{7}{|l|}{ Edad } \\
\hline $22-25$ años & 5 & $35.7 \%$ & 3 & $42.9 \%$ & 2 & $28.6 \%$ \\
\hline 26-30 años & 3 & $21.4 \%$ & 1 & $14.3 \%$ & 2 & $28.6 \%$ \\
\hline 30-35 años & 3 & $21.4 \%$ & 2 & $28.6 \%$ & 1 & $14.3 \%$ \\
\hline Mayor de 35 años & 3 & $21.4 \%$ & 1 & $14.3 \%$ & 2 & $28.6 \%$ \\
\hline \multirow[t]{2}{*}{ Total } & 14 & $100 \%$ & 7 & $100 \%$ & 25 & $100 \%$ \\
\hline & $\begin{array}{c}\text { M: } \\
31.36\end{array}$ & DT: 8.53 & $\begin{array}{c}\text { M: } \\
31.14\end{array}$ & DT: 9.51 & $\begin{array}{c}M: \\
31.57\end{array}$ & DT: 8.18 \\
\hline \multicolumn{7}{|l|}{ Titulación } \\
\hline $\begin{array}{l}\text { Diplomatura en Maestro } \\
\text { especialista en Educa- } \\
\text { ción Infantil }\end{array}$ & 8 & $57.1 \%$ & 4 & $57.1 \%$ & 4 & $57.1 \%$ \\
\hline $\begin{array}{l}\text { Técnico Superior en } \\
\text { Educación Infantil }\end{array}$ & 6 & $42.9 \%$ & 3 & $42.9 \%$ & 3 & $42.9 \%$ \\
\hline Total & 14 & $100 \%$ & 7 & $100 \%$ & 7 & $100 \%$ \\
\hline
\end{tabular}


Programa para la mejora de la sensibilidad (PMS) y habilidades de interacción del profesorado de Educación Infantil

Alexander Barandiaran Arteaga, Alexander Muela Aparicio, Elena lópez de Arana Prado, iñaki Larrea Hermida y Agurtzane Martínez Gorrotxategi

\begin{tabular}{lcccccc}
\hline Años de experiencia & & & & & & \\
\hline $1-5$ años & 7 & $50 \%$ & 4 & $57.1 \%$ & 3 & $42.9 \%$ \\
\hline 6-10 años & 4 & $28.6 \%$ & 2 & $28.6 \%$ & 2 & $28.6 \%$ \\
\hline $11-15$ años & 2 & $14.3 \%$ & 1 & $14.3 \%$ & 1 & $14.3 \%$ \\
\hline Más de 15 años & 1 & $7.1 \%$ & 0 & $\mathbf{0} \%$ & 1 & $14.3 \%$ \\
\hline Total & $\mathbf{1 4}$ & $\mathbf{1 0 0} \%$ & 7 & $\mathbf{1 0 0} \%$ & 7 & $\mathbf{1 0 0} \%$ \\
\hline & M: & DT: 5.65 & M: & DT: 4.95 & M: & DT: 6.58 \\
& 6.93 & & 6.14 & & 7.71 & \\
\hline
\end{tabular}

\section{Instrumentos}

Escala de Sensibilidad (NCKO Sensitivity Scale; De Kruif et al., 2007). La escala de sensibilidad tiene como objetivo evaluar la respuesta sensible de los maestros/as. En primer lugar, se realizan 3 grabaciones de 10 minutos de duración en el aula. Posteriormente, en función de la respuesta del maestro/a a las señales de los niños/as, se evalúa el grado de sensibilidad en una escala de 7 puntos, en un rango que varía de 1 a 7 . La puntuación más baja hace referencia a una respuesta muy insensible por parte del educador/a, mientras que la puntuación máxima refleja una alta sensibilidad. Se considera que el maestro/a presenta una alta sensibilidad cuando es capaz de proveer apoyo emocional a los niños/ as de forma continua, percibe adecuadamente las señales de los niños y responde a sus necesidades contingentemente. A continuación, se obtiene el promedio de las 3 observaciones. Esta escala de evaluación se basa en las escalas desarrolladas por Ainsworth y colaboradores para medir la sensibilidad de las figuras de apego en el contexto familiar (Ainsworth, Bell y Stayton, 1974).

El estudio de la fiabilidad entre los dos observadores que realizaron la evaluación se obtuvo mediante el modelo ANOVA mixto, acuerdo absoluto. El coeficiente de correlación intraclase promedio fue de .76 (en un rango que oscila entre 0,71 y 0,84$)$. La consistencia interna de la escala fue de .89 (en el pretest) y de .82 (en el postest).

Escala de Estimulación Verbal (NCKO Talking and Explaining Scale; De Kruif et al., 2007). La escala de estimulación verbal evalúa la capacidad de los maestros/as para estimular verbalmente a los niños/as. Se valora la frecuencia, el estilo y el contenido de la interacción verbal. La eva- 
Alexander Barandiaran Arteaga, Alexander Muela Aparicio, Elena lópez de Arana Prado,

luación se realiza examinando los mismos fragmentos utilizados para valorar la sensibilidad. Igualmente, se evalúa el grado de estimulación verbal en una escala de 7 puntos, en un rango que varía de 1 a 7 . A continuación, se obtiene el promedio de las 3 observaciones. Se considera que el maestro/a estimula verbalmente a los niños/as cuando hace un uso regular del lenguaje, ajustado al interés del niño/a y a su etapa evolutiva. El educador/a habla con mucha frecuencia al niño/a, le estimula a que ponga en palabras sus sentimientos e intenciones, y adapta el ritmo y el contenido de su discurso al desarrollo cognitivo y social del niño/a y a las actividades que se están llevando a cabo en ese momento. La puntuación más baja hace referencia a un perfil de educador/a que apenas habla o responde a los niños/as y que no estimula suficientemente la utilización del lenguaje. También se obtiene una muy baja puntuación cuando el educador/a se dirige a los niños/as principalmente de un modo negativo o solamente para corregir su comportamiento.

Se realizó un análisis de concordancia entre los dos observadores que realizaron la evaluación, obteniéndose un coeficiente de correlación intraclase promedio (ANOVA mixto, acuerdo absoluto) de .72 (en un rango que oscila entre .62 y .78). La consistencia interna de la escala fue de .86 (en el pretest) y de .84 (en el postest).

\section{Procedimiento}

La investigación se desarrolló en tres fases. En la primera de ellas, tras obtener el consentimiento para llevar a cabo el estudio tanto por parte de los centros educativos, las maestras y los padres de los menores, se recabaron una serie de datos sociodemográficos. A continuación se procedió a realizar las grabaciones en video de la práctica docente en las actividades seleccionadas tanto en el grupo de tratamiento como en el grupo de control. Se grabaron un total de 4 actividades. A saber, el momento de la recepción y acogida de los niños, una sesión de juego libre, una actividad guiada por la maestra y una sesión de psicomotricidad. Posteriormente, una semana antes de comenzar la intervención, se realizó la evaluación de la sensibilidad y la estimulación verbal de las maestras (medida pretratamiento).

En una segunda fase, se llevó a cabo el programa de formación para la mejora de la sensibilidad. En PMS tuvo una duración de 8 semanas 
Alexander Barandiaran Arteaga, Alexander Muela Aparicio, Elena lópez de Arana Prado,

y constó de 4 sesiones de intervención presencial formador-maestra. El grupo de profesionales encargado de la formación estuvo constituido por dos psicólogos y tres psicopedagogos. Todos ellos habían recibido formación especializada en la intervención para la mejora de la sensibilidad a través del video feedback en el ámbito escolar.

Por último, en una tercera fase, dos semanas después de la finalización del programa de formación, se volvieron a realizar las grabaciones de las mismas actividades y se evaluó la sensibilidad y la estimulación verbal, tanto en el grupo de intervención como en el grupo de control (medida postratamiento).

\section{Resultados}

En la tabla 2 se presentan las puntuaciones medias y las desviaciones típicas del grupo de tratamiento en el pretest y en el postest en la sensibilidad y la estimulación verbal de la educadora

Tabla 2. Puntuaciones medias y desviaciones típicas del grupo de tratamiento en la sensibilidad y la estimulación verbal antes y después de la intervención.

\begin{tabular}{lcccc}
\hline Variable & Evaluación & Media & Desv. típ & $N$ \\
\hline \multirow{2}{*}{ Sensibilidad } & Pretest & 3.86 & 1.46 & 7 \\
\cline { 2 - 5 } & Postest & 4.86 & 1.22 & 7 \\
\hline \multirow{2}{*}{ Estimulación del lenguaje } & Pretest & 4 & 1.15 & 7 \\
\cline { 2 - 5 } & Postest & 4.71 & 0.95 & 7 \\
\hline
\end{tabular}

Con objeto de analizar las diferencias existentes entre los valores de la sensibilidad y la estimulación verbal obtenidos por la educadora en el pretest y el postest, se aplicó la prueba de Wilcoxon. Los resultados pusieron de manifiesto que el Programa de Mejora de la Sensibilidad (PMS) ejercía una mejora estadísticamente significativa sobre la sensibilidad de la educadora $(Z=-2.496 ; p=.013)$. De acuerdo con este resultado, el tamaño del efecto asociado a la diferencia de rangos entre el pretest y el postest fue de gran magnitud $\left(\eta^{2}=.445\right)$.

En lo que se refiere a la estimulación del lenguaje, no se encontró una diferencia estadísticamente significativa. No obstante, el tamaño del efecto asociado a la diferencia de rangos entre el pretest y el postest fue de magnitud considerable $\left(\eta^{2}=.198\right)$. 
Programa para la mejora de la sensibilidad (PMS) y habilidades de interacción del profesorado de Educación Infantil

Alexander Barandiaran Arteaga, Alexander Muela Aparicio, Elena lópez de Arana Prado, Iñaki Larrea Hermida y Agurtzane Martínez Gorrotxategi

En las figuras 1 y 2 se presentan los patrones del grupo de tratamiento y del grupo de control en la sensibilidad y la estimulación del lenguaje.

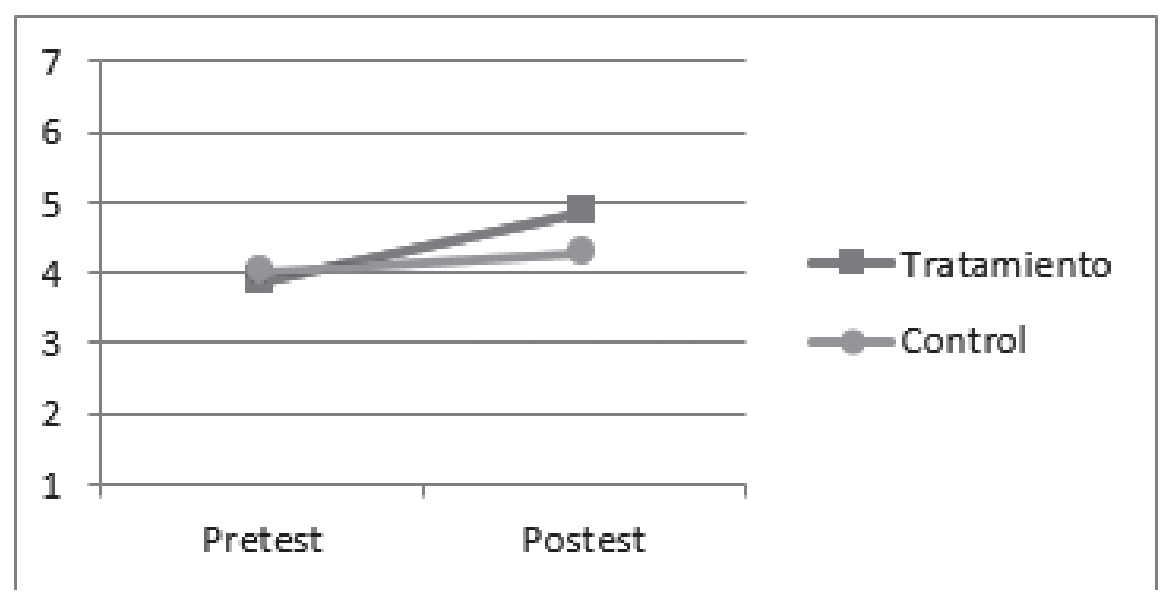

Figura 1. Puntuación promedio del grupo de tratamiento y del grupo de control en la sensibilidad antes y después de la formación.

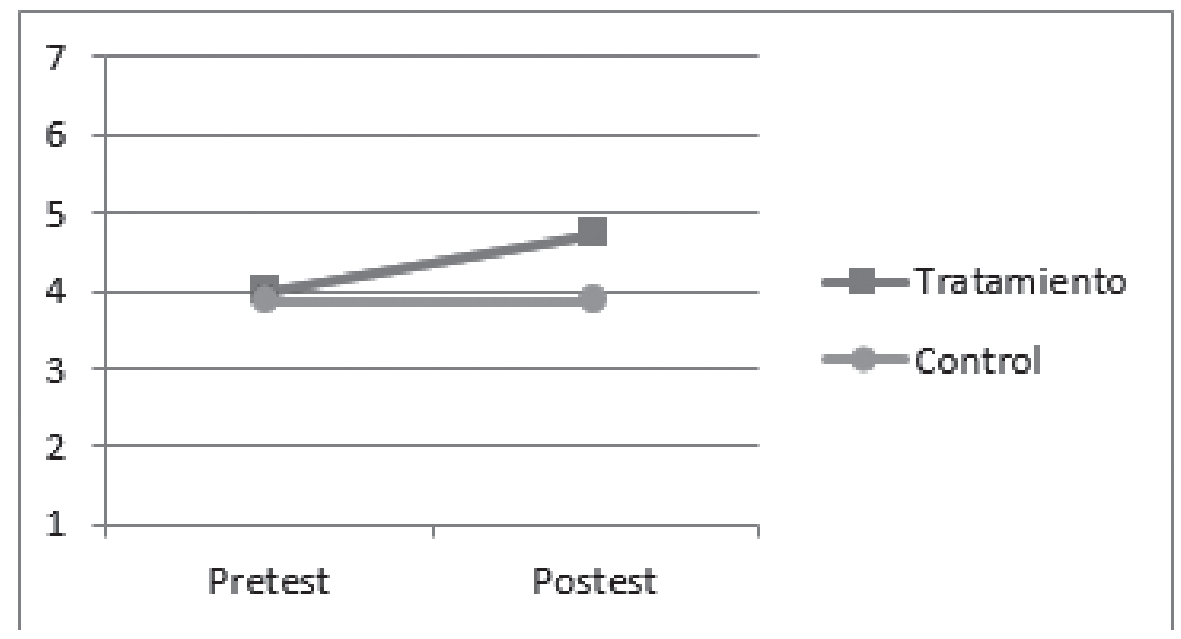

Figura 2. Puntuación promedio del grupo de tratamiento y del grupo de control en la estimulación del lenguaje antes y después de la formación.

Con el objetivo de examinar si existían diferencias entre el grupo de tratamiento y el grupo control en la sensibilidad y en la estimulación verbal antes y después de la intervención, se aplicó la prueba $U$ de MannWhitney, obteniéndose los resultados que se muestran en la tabla 3. 
Alexander Barandiaran Arteaga, Alexander Muela Aparicio, Elena lópez de Arana Prado, iñaki Larrea Hermida y Agurtzane Martínez Gorrotxategi

Tabla 3. Rangos promedio para el grupo de intervención y el grupo de control, $U$ de Mann-Whitney y tamaños del efecto asociados a las diferencias entre rangos.

\begin{tabular}{lccccccccc}
\hline Variables & $\begin{array}{c}\text { Evalua- } \\
\text { ción }\end{array}$ & $\begin{array}{c}\text { Grupo de } \\
\text { intervención }\end{array}$ & $\begin{array}{c}\text { Grupo de } \\
\text { control }\end{array}$ & \multicolumn{4}{c}{$\begin{array}{c}\text { U de Mann-Whit- } \\
\text { ney }\end{array}$} \\
\cline { 2 - 11 } & $\begin{array}{c}\text { Rango } \\
\text { promedio }\end{array}$ & $\mathbf{N}$ & $\begin{array}{c}\text { Rango } \\
\text { promedio }\end{array}$ & $\mathbf{N}$ & $\mathbf{U}$ & $\mathbf{Z}$ & Sig. & $\mathbf{\eta 2}^{*}$ \\
\hline Sensibilidad & Pretest & 7.57 & 7 & 7.43 & 7 & 24 & -0.067 & .946 & .001 \\
\cline { 2 - 12 } & Postest & 8.57 & 7 & 6.43 & 7 & 17 & -1.034 & .301 & .076 \\
\hline \multirow{2}{*}{$\begin{array}{l}\text { Estimulación } \\
\text { del lenguaje }\end{array}$} & Pretest & 7.64 & 7 & 7.36 & 7 & 23.5 & -0.135 & .892 & .001 \\
\cline { 2 - 10 } & Postest & 9.14 & 7 & 5.86 & 7 & 13 & -1.546 & .122 & .171 \\
\hline
\end{tabular}

*Según Cohen (1988), los valores de $\eta^{2}$ que oscilan entre .01 y .05 pueden ser considerados bajos, los que oscilan entre .06 y .13 pueden considerarse medios y los que superan el valor de .14 pueden ser considerados como valores altos.

La tabla III no muestra en el pretest diferencias estadísticamente significativas en las variables de sensibilidad y estimulación del lenguaje entre el grupo de tratamiento y el grupo de control. Además, cabe señalar que los tamaños del efecto asociados a dichas comparaciones fueron de pequeña magnitud. Por lo tanto, se puede concluir que ambos grupos son perfectamente comparables en las variables mencionadas, ya que no existían diferencias entre los grupos antes de introducir el tratamiento.

En lo que se refiere a las diferencias en el postest, no se hallaron diferencias estadísticamente significativas entre los rangos promedio del grupo de tratamiento y del grupo control. Sin embargo, los tamaños del efecto asociados a dichas comparaciones fueron de magnitud moderada $\left(\eta^{2}=.076\right)$ en el caso de la sensibilidad de la educadora y de gran magnitud $\left(\eta^{2}=.171\right)$ en la estimulación del lenguaje. Por lo tanto, siguiendo la recomendación de tomar como referencia los tamaños del efecto en contrastes no paramétricos con pocos sujetos (Cañadas, Borges, Sánchez y San Luis, 2000; Fritz, Morris y Richler, 2012), los resultados ponen de relieve que tras la intervención, las educadoras que participaron en la formación mostraban una mayor sensibilidad y estimulación del lenguaje que las educadoras del grupo de control.

\section{Conclusiones}

Podemos concluir, en base a los resultados obtenidos y tomando en consideración los objetivos planteados en la presente investigación, que 
Alexander Barandiaran Arteaga, Alexander Muela Aparicio, Elena lópez de Arana Prado,

tras someterse al PMS, el profesorado presenta una mayor sensibilidad para proveer apoyo emocional a los niños/as de forma continua, percibir adecuadamente las señales de los niños y responder a sus necesidades contingentemente. Asimismo, los maestros/as muestran un incremento de las conductas de estimulación verbal. Además, en comparación con los docentes que no habían recibido el tratamiento, presentan una mayor puntuación en las variables de sensibilidad y estimulación verbal. Por lo tanto, estos datos apuntan a que la aplicación del PMS puede resultar un programa de formación útil para mejorar las habilidades de interacción de los educadores/as de Educación Infantil. Estos resultados coinciden con los de otras investigaciones recientes que han encontrado resultados similares (Elicker et al., 2008; Fukkink y Tavechhio, 2010; Pianta et al., 2008).

Partiendo de esta conclusión, queremos destacar la importancia de contar con un desarrollo profesional del profesorado de Educación Infantil dirigido a mejorar las habilidades de interacción del profesorado, y más concretamente, la sensibilidad y la capacidad de estimulación verbal. Tal y como postula la teoría del apego (Bowlby, 1969), y han evidenciado empíricamente diversos estudios realizados en el ámbito familiar (Bernier, Carlson y Whipple, 2010; Simó y D'Ocon, 2011) y escolar (Barandiaran et al., 2015; Elicker et al., 2008et al., 2008), la experiencia continuada de sensibilidad por parte de los cuidadores, a través del tiempo y de los contextos, proporciona al niño/a una base segura, fundamental para un adecuado desarrollo socioemocional y cognitivo.

La escuela no puede ser una excepción en este sentido; al contrario, debe ser un factor clave en este proceso (Sabol y Pianta, 2012; Spilt, Koomen, Thijs y van der Leij, 2012). Los niños y las niñas ingresan cada vez más pronto en esta institución y cada vez pasan mayor tiempo en ella (OECD, 2013, 2014), por lo que es especialmente relevante que experimenten un trato sensible que les ayude a abordar con éxito la transición entre el contexto familiar y el escolar, así como las complejas y continuadas experiencias relacionales con otros niños/as, personas adultas, espacios y materiales, lo que a la postre incrementará las posibilidades de un adecuado desarrollo cognitivo, lingüístico y socioemocional. Por consiguiente, creemos esencial que los maestros/as de Educación Infantil adquieran en su desarrollo profesional una apropiada capacitación en la conducta de sensibilidad. Es decir, la formación en la percepción e interpretación adecuada de las señales de los niños/as, así 
Alexander Barandiaran Arteaga, Alexander Muela Aparicio, Elena lópez de Arana Prado,

como en responder de forma contingente a dichas señales, lo que a su vez, exige mostrarse emocionalmente disponible y atento a las necesidades infantiles.

Por otra parte, la estimulación verbal, tan ligada a la respuesta sensible, es también un elemento de vital importancia, puesto que la alta estimulación verbal del educador/a en la primera infancia se ha asociado a un mayor desarrollo cognitivo y del lenguaje (Howes et al., 2008; NICHD, 2000; NICHD y Duncan, 2003).

Una segunda reflexión que inspira este estudio hace referencia al tipo de formación que se ha de proporcionar. Existe un consenso general en considerar la formación del docente como un factor clave para disponer de una alta calidad de Educación Infantil (García-Ruiz y Castro, 2012; Han, 2014; OECD, 2012; Rodríguez-Martínez y Díez, 2014; Zaslow y Martinez-Beck, 2005). Por lo tanto, al maestro/a se le ha de ofrecer la oportunidad de favorecer su desarrollo profesional, para lo que es indispensable la implicación institucional. Un aspecto central de la política europea de educación no es el hecho de invertir en la formación del profesorado de Educación Infantil, puesto que esto ya se hace, sino en qué cantidad, en qué nivel se está realizando y si esta formación está adaptada a las necesidades docentes de cada ciclo educativo (OECD, 2012). Zaslow y sus colaboradores (Zaslow y Martinez-Beck, 2005; Zaslow et al., 2010), en su extensa revisión sobre la investigación del desarrollo del profesorado de Educación Infantil, concluyen que el desarrollo profesional ha de enfocarse al análisis de la práctica, en combinación con otros cursos especializados que estén directamente relacionados con las "buenas prácticas". Además, inciden en la importancia de fomentar las formaciones de carácter grupal. A este respecto, a la luz de los resultados obtenidos en el presente estudio, también se hace hincapié en el beneficio que se obtiene de un tipo de formación basado en el análisis de la práctica y desempeño del puesto de trabajo (García-Ruiz y Castro, 2012; Pérez Ferra, 2013; González Calvo y Barba, 2014), pero al igual que otros estudios (Fukkink et al., 2011), se acentúa la importancia de la utilización de la metodología del video feedback.

Por otra parte, en lo que se refiere a España, existe una tradición de desarrollo profesional en el sector. Sin embargo, cabe señalar que el alcance del desarrollo profesional en Educación Infantil es muy inferior en el primer ciclo (Pineda, Moreno, úcar y Belvis, 2008). Mientras el ciclo de 3-6 años muestra un porcentaje de tradición de formación más 
Alexander Barandiaran Arteaga, Alexander Muela Aparicio, Elena lópez de Arana Prado,

alto de lo esperado, el ciclo 0-3 obtiene un porcentaje inferior. Además, cabe señalar que las posibilidades de participación en el desarrollo profesional son menores ya que, en general, las condiciones de trabajo son más precarias (Pineda et al., 2008). A nuestro parecer, se han de realizar esfuerzos por garantizar el acceso al desarrollo profesional del personal docente del primer ciclo de Educación Infantil y fortalecer la calidad de la misma.

\section{Limitaciones del estudio y prospectiva}

No queremos finalizar el trabajo sin señalar algunas de sus limitaciones. La primera de ellas se relaciona con la imposibilidad de utilizar la asignación aleatoria para la formación de los grupos de intervención y de control dado que fueron los centros escolares quienes decidieron qué educadores/as debían recibir el programa de formación. Aunque ello reduce la validez interna del estudio, se utilizaron diversas variables de emparejamiento con el objetivo de que los grupos fuesen comparables.

En segundo lugar, cabe citar el reducido tamaño de la muestra utilizada, lo que disminuye la potencia estadística y la capacidad para generalizar los resultados obtenidos. Por lo tanto, en el futuro ha de replicarse el estudio con un mayor número de sujetos.

Por último, para profundizar en el estudio de la influencia del PMS sobre las habilidades de interacción de los maestros y las maestras de Educación Infantil, además de la muestra estudiada, debería examinarse el profesorado del segundo ciclo de Educación Infantil y contar también con participantes de género masculino y diversidad en los años de experiencia profesional docente. También se debería disponer de la participación de profesores de escuelas públicas y no solamente ubicadas en el ámbito urbano.

A pesar de estas limitaciones, los resultados obtenidos permiten afirmar que el PMS es un tipo de formación que puede ser muy efectivo para mejorar las habilidades de interacción de los docentes de Educación Infantil. 
Programa para la mejora de la sensibilidad (PMS) y habilidades de interacción del profesorado de Educación Infantil

Alexander Barandiaran Arteaga, Alexander Muela Aparicio, Elena lópez de Arana Prado, Iñaki Larrea Hermida y Agurtzane Martínez Gorrotxategl

\section{Referencias Bibliográficas}

Ainsworth, M. D. S., Bell, S. M. \& Stayton, D. J. (1974). Infant-mother attachment and social development: Socializations as a product of reciprocal responsiveness to signals. En M. P. M. Richards (Ed.), The integration of a child into social world (pp.99-135). London: Cambridge University Press.

Ancheta, A. (2012). El derecho a la educación y atención de la primera infancia en la Europa actual. Educatio Siglo XXI, 30, 129-148.

Barandiaran, A., Muela, A., López de Arana, E., Larrea, I., \& Vitoria, J. R. (2015). Conducta de exploración, bienestar emocional y calidad del cuidado en Educación Infantil. Anales de Psicología, 31, 570-578.

Bautista, A. (2013). Funciones del vídeo en la formación del profesorado para una educación intercultural. Educatio Siglo XXI, 31, 255-268.

Belsky, J. (2006). Effects of child development in the USA. In J. J. van Kuyk (Ed.) The quality of early childhood education (pp. 23-32). Arnheim: Cito.

Bernier, A., Carlson, S. M., \& Whipple, N. (2010). From external regulation to self-regulation: Early parenting precursors of young children's executive functioning. Child Development, 81, 326-339.

Burchinal, M. R., Howes, C., Pianta, R., Bryant, D., Early, D., Cliffors, R., \& Barbarin, B. (2008). Predicting child outcomes at the end of kindergarten from the quality of pre-kindergarten teacher-child interactions and instructions. Applied Developmental Science, 12, 140-153.

Burchinal, M. R., Roberts, J. E., Riggins, R., Zeisel, S. A., Neebe, E., \& Bryant, D. (2000). Relating quality of center-based child care to early cognitive and language development longitudinally. Child Development, 71, 339-357.

Bowlby, J. (1969). Attachment and loss: Vol. 1. Attachment. Nueva York: Basic Books. [Trad. cast. de M. Valcarce, El apego y la pérdida: Vol. 1. El apego. Barcelona: Paidós, 1998].

Calderón, A., \& Martínez, D. (2014). La formación permanente del profesorado de educación física. Propuesta de enseñanza del modelo de educación deportiva. Revista de Educación, 363, 128-153.

Cañadas, I., Borges, A., Sánchez, A., \& San Luis, C. (2000). Estudio de la potencia de los contrastes de medias con dos y tres grupos con tamaño de efecto pequeño y en condiciones de no normalidad y homo-heterocedasticidad. Psicothema, 12, 114-116.

Clarke-Stewart, A., \& Allhusen, V. D. (2005). What we know about childcare. Cambridge, MA: Harvard University Press.

Cohen, J. (1988). Statistical power analysis for the behavioral sciences. Hillsdale: Lawrence Earlbaum Associates

De Kruif, R. E. L., Vermeer, H. J., Fukkink, R. G., Riksen-Walraven, J. M. A., Tavecchio, L. W. C., van Ijzendoorn, M. H., \& van Zeijl, J. (2007). The national study on childcare quality: Final report project $O$ and 1. Amsterdam: NCKO.

De Schipper, E. J., Riksen-Walraven, J. M., \& Geurts, S. A. E. (2006). Effects of childcaregiver ratio on the interactions between caregivers and children in child-care centers: an experimental study. Child Development, 77, 861-874. 
Programa para la mejora de la sensibilidad (PMS) y habilidades de interacción del profesorado de Educación Infantil

Alexander Barandiaran Arteaga, Alexander Muela Aparicio, Elena lópez de Arana Prado, Iñaki Larrea Hermida y Agurtzane Martínez Gorrotxategl

Elicker, J., Georgescu, O., \& Bartsch, E. (2008). Increasing the sensitivity of infant toddler child care providers; applying the VIPP approach. In F. Juffer, M. J. Bakermans-Kranenburg y M. H. van IJzendoorn (Eds.), Promoting positive parenting: An attachmentbased intervention (pp. 155-170). Hillsdale, NJ: Lawrecne Erlbaum.

Fritz, C. O., Morris, P. E., \& Richler, J. J. (2012). Effect size estimates: current use, calculations, and interpretation. Journal of Experimental Psychology, 141, 2-18.

Fukkink, R. G. (2008). Video feedback in widescreen: A meta-analylis of family programs. Clinical Psychology Review, 28, 904-916.

Fukkink, R. G., \& Lont, A. (2007). Does training matter? A meta-analysis and review of caregiver training studies. Early Childhood Research Quarterly, 22, 294-311.

Fukkink, R. G., \& Tavecchio, L. W. C. (2010). Effects of video Interaction Guidance on early childhood teachers. Teaching and Teacher Education, 26, 1652-1659.

Fukkink, R. G., Trienekens, N, \& Kramer, J. C. (2011). Video feedback in education and training: putting learning in the picture. Educational Psychology Review, 23, 45-63.

García-Ruiz, R., \& Castro, A. (2012). La formación permanente del profesorado basada en competencias. Estudio exploratorio de la percepción del profesorado de educación infantil y primaria. Educatio Siglo XXI, 30, 297-322.

González Calvo, G., \& Barba, J. J. (2014). Formación permanente y desarrollo de la identidad reflexiva del profesorado desde las perspectivas grupal e individual. Profesorado. Revista de Currículum y Formación del Profesorado, 18, 397-411.

Han, H. S. (2014). Supporting early childhood teachers to promote children's social competence: components for the best professional development practices. Early Childhood Education, 42, 171-179.

Hamre, B., Hatfield, B., Pianta, R., \& Jamil, F. (2014). Evidence for general and domainspecific elements of teacher-child interactions: associations with preschool children's development. Child Development, 85, 1257-1274.

Howes, C., Burchinal,M., Pianta, R., Bryant, D., Early., D., Clifford, R., \& Barbarin, O. (2008). Ready to learn? Children's pre-academic achievement in pre-Kindergarden programs. Early Childhood Research Quarterly, 23, 27-50.

Imbermon, F. (1994) La formación y el desarrollo profesional del profesorado. Hacia una nueva cultura profesional. Grao: Barcelona.

Imbermón, F (2005). Vivencias de maestros y maestras. Compartir desde la práctica educativa. Grao: Barcelona.

Imbermón, F (2007) La formación permanente del profesorado. Nuevas ideas para formar en la innovación y en el cambio. Grao: Barcelona.

Juffer, F., Bakermans-Kranenburg, M. J., \& van IJzendoorn, M. H. (2008). Promoting positive parenting: An attachment based intervention. Mahwah, NJ: Erlbaum.

Lera, M. J. (2007). Calidad en la Educación Infantil. Revista de Educación, 343, 301-323.

Manzanares, A., \& Galván-Bovaira, M. J. (2012). La formación permanente del profesorado de Educación Infantil y primaria a través de los centros de profesores. Un modelo de evaluación. Revista de Educación, 359, 431-455.

Marcelo, C. (2009). La evaluación del desarrollo profesional docente: de la cantidad a la calidad. Revista Brasileira de Formação, 1, 43-70. 
Programa para la mejora de la sensibilidad (PMS) y habilidades de interacción del profesorado de Educación Infantil

Alexander Barandiaran Arteaga, Alexander Muela Aparicio, Elena lópez de Arana Prado, Iñaki Larrea Hermida y Agurtzane Martínez Gorrotxategl

Marcelo, C. (2011). La profesión docente en momentos de cambio. ¿Qué nos dicen los informes internacionales? Participación Educativa, 16, 49-68.

Marcelo, C., \& Vaillant, D. (2009). Desarrollo profesional docente ¿Cómo se aprende a enseñar? Madrid: Narcea.

Mashburn, A., Pianta, R., Hamre, B., Downer, J., Barbarin, O., Bryant, D., Burchinal, M., Early, D., \& Howes, C. (2008). Measures of pre-k quality and children's development of academic, language and social skills. Child Development, 79, 732-749.

NICHD Early Child Care Research Network (2000). Characteristics and quality of child care for toddlers and preschoolers. Applied Developmental Science, 3, 116-135.

NICHD Early Child Care Research Network, \& Duncan, G. J. (2003). Modeling the impacts of child care quality on children's preschool cognitive development. Child Development, 74, 1454-1475.

NICHD Early Child Care Research Network (2005). Child care and child development: Results from the NICHD Study of Early child Care and Youth Development. The Guildford Press: Nueva York.

Ochaita, E., \& Espinosa, M. A. (2001). Los derechos de la infancia desde la perspectiva de las necesidades. Educatio Siglo XXI, 30, 25-46.

OECD (2012). Starting strong III. A quality toolbox for early childhood education and care. Paris: OECD Publications. Disponible en http://www.oecd.org/edu/school/startingstrongiii-aqualitytoolboxforearlychildhoodeducationandcare.htm (Consultado 5/02/2014).

OECD (2013). Education at a glance 2013. OECD indicators. Paris: OECD Publications. Disponible en http://www.oecd.org/edu/eag2013\%20\%28eng\%29--FINAL\%20 20\%20June\%202013.pdf (Consultado 5/02/2014).

OECD (2014). Education policy outlook. Spain. Paris: OECD Publications. Disponible en http://www.oecd.org/edu/EDUCATION\%20POLICY\%20OUTLOOK\%20SPAIN_ EN.pdf (Consultado 20/05/2014).

Peisner-Feinberg, E. S., Burchinal, M. R., Clifford, R. M., Culkin, M. L., Howes, C., Kagan, S. L., \& Yazejian, N. (2001). The relation of preschool child care quality to children's cognitive and social developmental trajectories through second grade. Child Development, 72, 1534-1553.

Pérez Ferra, M. (2013). La actitud indagadora del profesor: un proceso para desarrollar competencias en los docentes. Profesorado. Revista de Currículum y Formación del Profesorado, 17, 58-72.

Phillips, D. A., \& Lowenstein, A. E. (2011). Early care, education, and child development. Annual Review of Psychology, 62, 483-500.

Pianta, R. C., Mashburn, A. J., Downer, J. T., Hamre B. K., \& Justice, L. (2008). Effects of web mediated professional development resources on teacher-child interactions in pre-kindergarden classrooms. Early Childhood Research Quarterly, 23, 431-451.

Pineda, P., Moreno. M. V., Úcar, J., \& Belvis, E. (2008). Derecho a la calidad de la formación permanente en el sector de la Educación Infantil en España. Revista de Educación, 347, 101-126.

Orden ECI/3854/2007: verificación de los títulos universitarios oficiales que habiliten para el ejercicio de la profesión de Maestro en Educación Infantil. BOE, 312 (2007). 
Programa para la mejora de la sensibilidad (PMS) y habilidades de interacción del profesorado de Educación Infantil

Alexander Barandiaran Arteaga, Alexander Muela Aparicio, Elena lópez de Arana Prado, Iñaki Larrea Hermida y Agurtzane Martínez Gorrotxategl

Disponible en http://www.boe.es/boe/dias/2007/12/29/pdfs/A53735-53738.pdf (Consultado 31/04/2015).

Rodríguez-Martínez, C., \& Díez, E. J. (2014). Conocimiento y competencias básicas en la formación inicial de maestras y maestros. Profesorado. Revista de Currículum y Formación del Profesorado, 18, 383-396.

Ruzek, E., Burchinal, M. R., Farkas, G., \& Duncan, G. J. (2014). The quality of toddler child care and cognitive skills at 24 months: propensity score analysis results from the SCLS-B. Early Childhood Quarterly, 29, 12-21.

Sabol, T. J., \& Pianta, R. C. (2012). Recent trends in research on teacher-child relationships. Attachment y Human Development 14, 213-231.

Simó, S., \& D'Ocon, A. (2011). La estructura temporal de la experiencia de sensibilidad maternal: su efecto sobre el desarrollo cognitivo y emocional infantil. Infancia y Aprendizaje, 34, 481-493

Spilt, J. L., Koomen, H. M. Y., Thijs, J. T., \& van der Leij, A. (2012). Supporting teachers' relationships with disruptive children: the potential of relationship-focused reflection. Attachment y Human Development 14, 305-318.

Tripp, T. R., \& Rich, P. J. (2012). The influence of video analysis on the process of teacher change. Teaching and Teacher Education, 28, 728-739.

van Vonderen, A., Didden, R., \& Beeking, F. (2012). Effectiveness of instruction and video feedback on staff's trainer behavior during one-to-one training children with severe intellectual disability. Research in Developmental Disabilities, 31, 283-290.

Vandell, D. L., Belsky, J., Burchinal, M., Vandergrift, N., \& Steinberg, L. (2010). Do effects of early child care extend to age 15 years? Results from the NICHD study of early child care and youth. Child Development, 81, 737-756.

Virmani, E. A., \& Ontai, L. L. (2010). Supervision and training in child care: does reflective supervision foster caregiver insightfulness? Infant Mental Health Journal, 31, 16-32.

Zabalza, M. A. (1996). Calidad en la Educación Infantil. Madrid: Narcea.

Zabalza, M. A., \& Zabalza, M. A. (2011). La formación del profesorado de Educación Infantil. CEE Participación Educativa, 16, 103-113.

Zaslow, M., Anderson, R., Redd, Z. Wessel, J., Tarullo, L., \& Burchinal, M. (2010). Quality Dosage, Thresholds, and Features in Early Childhood Settings: A Review of the Literature, OPRE 2011-5. Washington, DC: U.S. Department of Health and Human Services, Administration for Children and Families, Office of Planning, Research and Evaluation. Disponible en http://www.acf.hhs.gov/sites/default/files/opre/quality_review_0.pdf (Consultado 21/05/2014).

Zaslow, M., \& Martinez-Beck, I. (2005). Critical issues in early childhood professional development. Baltimore: Brookes. 